\title{
An Improved 6LoWPAN Hierarchical Routing Protocol
}

\author{
Li Xue \\ Key Laboratory of Broadband Wireless Communication \\ and Sensor Network Technology \\ Nanjing University of Posts and Telecommunications \\ NanJing, China \\ lixue7168@163.com
}

\author{
Sun Zhixin \\ Key Laboratory of Broadband Wireless Communication \\ and Sensor Network Technology \\ Nanjing University of Posts and Telecommunications \\ NanJing, China \\ sunzx@njupt.edu.cn, szx630@sina.com
}

\begin{abstract}
IETF 6LoWPAN working group is engaged in the IPv6 protocol stack research work based on IEEE802.15.4 standard. In this working group, the routing protocol is one of the important research contents. In the 6LoWPAN, HiLow is a wellknown layered routing protocol. This paper puts forward an improved hierarchical routing protocol GHiLow by improving HiLow parent node selection and path restoration strategy. GHiLow improves the parent node selection by increasing the choice of parameters. Simutaneously, it also improves path recovery by analysing different situations to recovery path. Therefore, GHiLow contributes to the ehancement of network performance and the decrease of network energy consumption.
\end{abstract}

Keywords-6LoWPAN; HiLow; path recovery; parent node selection

\section{INTRODUCTION}

6LoWPAN [1] (IPv6 over Low power Wireless Personal Area Networks) technology through the introduction of adaptation layer between the network layer and data link layer to realize the underlying network based on IEEE802.15.4 communications protocol and internet seamless link to each other based on IPv6 protocol, thus it provides reliable network protocol for Internet of Things large-scale applications. Usually there are a lot of devices being deployed in 6LoWPAN, and these devices have a limited capacity, and the nodes' burden of the presence of routing tables and EUI-64-bit address to store in 6LoWPAN.Therefore, HiLow hierarchical mechanism has been proposed. HiLow [2] is a kind of hierarchical routing protocol based on the short address assigned dynamically in 6LoWPAN.HiLow doesn't need to store the routing table and neighbor discovery by flooding process. But its shortcomings such as the parent node selection and path recovery limit its application and development.

Thanks to the flaws of the parent node selection strategy and the path recovery strategy, the paper puts forward an improved hierarchical routing protocol GHiLow. In the parent node selection: GHiLow is based on the existing literature to consider the depth of the node, node's residual energy, and the number of node's children and so on, considering the factor of the order confirmation message arrives .And the situation that the candidate parent node is a leaf node is analyzed. It allows the node to select a better parent node, enhance the performance of the network, and prolong the survival time of network. In path recovery: Firstly, all the lower-depth nodes of failure node in accordance with the order from the bottom up in order to detect the existence of the destination node within its communication range .If the destination node is detected and the number of the destination node's children under the limit, the node breaks away from the original parent node and associated with the destination node. When all the lower-depth nodes of failure node complete detection using existing the child nodes as root to develop tree ,only the subtree root node choose the new parent node to join the non-failing parts of the tree. The path recovery effectively reduces the energy consumption of the network.

\section{RELATED WORK}

HiLow uses 6LoWPAN characteristics of dynamically configuring node's 16 bit short address to realize the initialization configuration. When a node wants to join the 6LoWPAN network, it must detect whether existing a 6LoWPAN network within its communication range .If the node doesn't detect network, the node will automatically act as a new 6LoWPAN network coordinator node and assign a short address of value is 0 for itself; If the detection is successful, the node associated with the parent node and can receive a 16-bit short addresses from the parent node. The formula of address assignment as follows:

$$
F C=M C * A P+N(O<N<=M C)
$$

, where $M C$ is the maximum number of child nodes that a parent can have, $A P$ is the address of parent node, $N$ is the Nth child node of parent node[3].

Nowadays there are a lot of researches based on HiLow hierarchical routing protocol. In the parent node selection, someone [4] chooses the node that has the least child node as its parent. But it doesn't take into account the problem of limited energy of nodes. Someone else [5] think to make the parent node selection depending on the depth of parent, the remaining energy and so on. But it ignores the impact of the number of children nodes .Someone others [6] proposed EHiLow, E-HiLow fully considers the node depth, residual energy and the number of child and so on. It selects the node with most average remaining energy and minimum depth to be 
its parent node. But the formula does not consider the situation that the candidate stepfather nodes are leaf nodes.

In HiLow hierarchical routing protocol path recovery, someone [7] suggests selecting an upstream node to be the agent of packets forwarding for the failure node's children. But it does not take into account the changes to all the lower-depth nodes of failure node. Someone others [8] proposed a new path recovery SPN algorithm, which looks for a new parent node for the child nodes of failure node and assigns a new 16 bit short address. However, it does not specify how to choose when appearing multiple candidate stepfathers.

Aimed at the above problems, this paper puts forward an improved hierarchical routing protocol--------GHiLow. In the parent node selection, GHiLow gives full consideration to the node depth, residual energy, the number of node's children and the order of confirmation message arrives to choose the parent node, and it takes into account the situation that the candidate parent node is a leaf node. GHiLow enables the nodes to better for the choice of the parent node.

In path recovery:

- All the lower-depth nodes of failure node in accordance with the order from the bottom up in order to detect the existence of the destination node within its communication range .If the destination node is detected and the number of the destination node's children under the limit, the node breaks away from the original parent node and associated with the destination node. It effectively reduces nodes communication process energy consumption.

- In the process of path recovery, when there are many candidate stepfather nodes according to the parent node selection method to select the parent node.

- The child node of new recovery node will drill update its own offspring nodes' 16 short address.

\section{GHILOW}

In GHiLow, when a new node wants to join 6LoWPAN network, the new node according to the node depth, residual energy, the number of node's children and the order of confirmation message arrives to choose the parent node. And GHiLow consider the situation that candidate parent node's children count is zero.

In GHiLow, when a node fails, all the lower-depth nodes of failure node in accordance with the order from the bottom up in order to detect the existence of the destination node within its communication range .If the destination node is detected and the number of the destination node's children under the limit, the node breaks away from the original parent node and associated with the destination node. And the node can receive a new 16-bit short address. When all the lower-depth nodes of failure node complete detection using existing the child nodes as root to develop tree, only the subtree root node choose the new parent node to join the non-failing parts of the tree. After joining, all subtree nodes will receive new 16 bit short address.
GHiLow assumes that each node can know the maximum number of child nodes, and its own depth.

\section{A. Parent Node Selection}

Someone [6] fully considers the node depth, residual energy and the number of node's child and so on. It selects the node with most average remaining energy and minimum depth to be its parent node.

$$
N p \Leftarrow \max \left\{\frac{p 1}{m 1^{*}\left(M C^{D 1}\right)}, \frac{p 2}{m 2 *\left(M C^{D 2}\right)}, \ldots, \frac{p_{n}}{m_{n} *\left(M C^{D_{n}}\right)}\right\}
$$

, where $N_{p}$ is the parent node, ${ }_{M C}$ is the maximum number of child nodes that a parent can have (the value is 4 ), $p_{i}$ is the remaining energy for the node $i, m_{i}$ is the number of child node for the node $i, D_{i}$ is the depth for the node $i$.

The defects of formula (2) as follows:

- When a node detects two candidate parent nodes have same node depth, residual energy and the number of node's child, formula (2) can't help the node to select the parent node. As shown in figure 1 , when $n 1$ node wants to join the 6LoWPAN network, detecting 5 and 9 two candidate parent nodes. Obviously two candidate parent nodes have same node depth and the number of node's child, if they have same residual energy, formula (2) can't help the n1 node to select the parent node.

- When the candidate parent nodes are leaf nodes, mi value is 0 , the formula (2) fails. As shown in figure 1, when the $\mathrm{n} 2$ node wants to join the 6LoWPAN network, detecting 21 and 37 two candidate parent nodes. But we can't use the formula (2) to select the parent node. When the denominator is zero, the formula (2) fails.

- When node detects the candidate parent nodes both the number of child nodes is zero and the number of child nodes isn't zero, because if the number of the child nodes is zero, the formula will be fail, so we can only choose the candidate parent node whose the number of child nodes is not zero, ignoring the candidate parent nodes whose the number of child nodes is zero. As shown in figure 1 , when the $\mathrm{n} 3$ node wants to join the 6LoWPAN network, detecting 3 and 4 two candidate parent nodes. But we can't use the formula (2) to select the 4 parent node. When the denominator is zero, the formula (2) fails. So $n 3$ chooses node 3 directly to its parent. In fact, the choice of node 4 is more conducive to prolong the survival time of the network. 


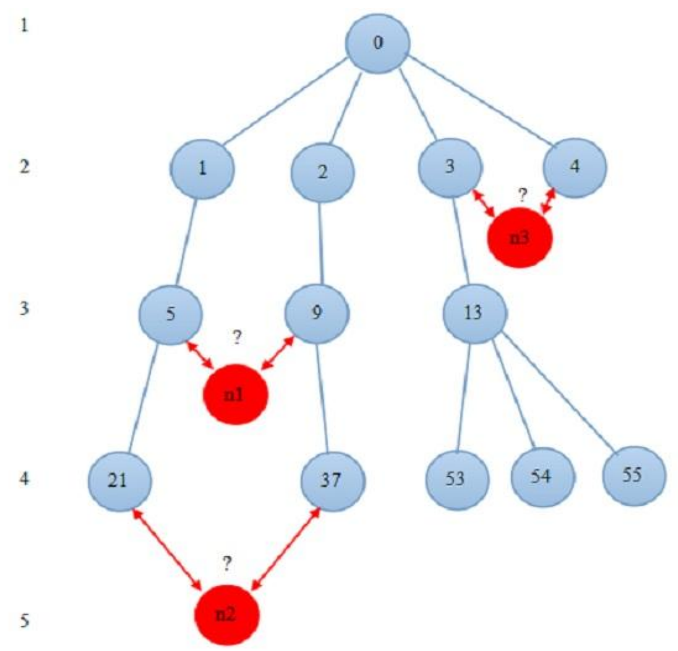

Figure 1. Parent node selection.

Aiming at the defects of formula (2), the paper considers the order of confirmation message arrives as one of parent node selection criteria to improve it. When a node wants to join the 6LoWPAN network, detecting the existence of linkable effective candidate parent nodes (the number of child nodes should be less than the MC) within its communication range, then according to the number of child nodes respectively using the following formula.

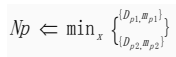

$$
\begin{aligned}
& 0<m_{i}<M C \text { : } \\
& \left\{D_{p 1}, m_{p 1}\right\} \Leftarrow \max \left\{\frac{p_{1}}{m_{1} *(M C)^{D_{1}} * \ln \left(s_{1}+10\right)}, \frac{p_{2}}{m_{2} *(M C)^{D_{2}} * \ln \left(s_{2}+10\right)}, \ldots, \frac{p_{n}}{m_{n} *(M C)^{D_{n}} * \ln \left(s_{n}+10\right)}\right. \\
& m_{i}=0 \text { : } \\
& \left\{D_{p 2}, m_{p 2}\right\} \Leftarrow \max \left\{\frac{p_{1}}{D_{1} * \ln \left(s_{1}+10\right)}, \frac{p_{2}}{D_{2} * \ln \left(s_{2}+10\right)}, \ldots, \frac{p_{n}}{D_{n} * \ln \left(s_{n}+10\right)}\right\}
\end{aligned}
$$

, where ${ }^{N_{p}}$ is the parent node, ${ }^{M C}$ is the maximum number of child nodes that a parent can have. ${ }^{p_{i}}$ is the remaining energy for the node $i, m_{i}$ is the number of child node for the node $i, \boldsymbol{D}_{i}$ is the depth for the node $i, S_{i}$ is the order of confirmation message arrives for the node $i, D_{p l}$ and ${ }^{m_{p l}}$ indicate the depth of the parent node and the number of child nodes, when the number of the candidate parent node's child node isn't zero, ${ }^{D^{2}}$ and ${ }^{m_{p 2}}$ indicate the depth of the parent node and the number of child nodes, when the number of the candidate parent node's child node is zero.

$$
\begin{aligned}
& x \text { structure as follows: } \\
& 1 \text { if } D_{p 1}:=D_{p 2} \\
& 2 \quad x=D \\
& 3 \text { else } x=m
\end{aligned}
$$

By selecting classification we can get two candidate parent nodes. When they don't have the same depth, the paper will consider depth as criterion and choose the candidate parent node that its depth is lower. If they have the same depth, the paper will consider the number of child nodes as criterion and chooses the candidate parent node that its number of child nodes is less.

This formula selects the node with most average remaining energy, minimum depth and the confirmation message to arrive early to be its parent node. According to the number of child nodes, the node chooses parent node when it receives confirmation messages from more than one candidate parent node. By selecting classification the node according to depth of candidate parent nodes to do choice. If they have the same depth, the node chooses the candidate parent node that its number of child nodes is less. Due to the formula considers more parameters and parent node's own conditions, it allows the node to better choose the parent node and prolong the survival time of network.

\section{B. Path Recovery}

The re-access of all lower nodes of failed node has a great influence on the performance of the network, especially in the case of failed node depth is lower. Therefore, it need to be carefully considered on the selection of the stepfather node and the new address updating of all lower nodes under failed node. Take into account the stepfather node load, performance, and the influence of the energy consumption of the network in the process of communication. So this article presents a more optimal path recovery method after considering the above factors.

When a node fails, all the lower-depth nodes of failure node in accordance with the order from the bottom up in order to detect the existence of the destination node within its communication range .If the destination node is detected and the number of the destination node's children under the limit, the node breaks away from the original parent node and associated with the destination node. And the node can receive a new 16-bit short address. If the destination node isn't detected, the node keeps the existing state of associated. It can effectively reduce the energy consumption in the communication process .When all the lower-depth nodes of failure node complete detection using existing the child nodes as root to develop tree, only the subtree root node choose the new parent node to join the non-failing parts of the tree. If there are many candidate stepfather nodes, the paper uses the parent node selection method in 3.1 to choose. After joining, all subtree nodes will receive new 16 bit short address. The child node of new recovery node will drill update its own offspring nodes'16 short address according to the formula (1). 


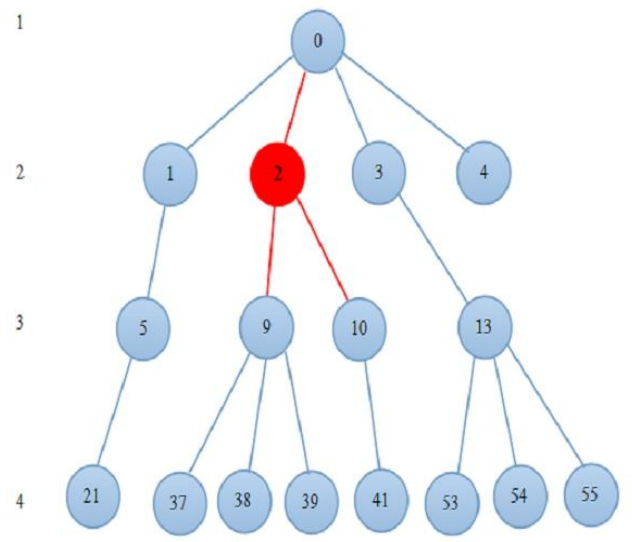

Figure 2. Path recovery.

As shown in figure 2, when the node 2 fails, all the lowerdepth nodes $\{9,10,37,38,39,41\}$ of node 2 .All the lower-depth nodes in accordance with the order from the bottom up in order to detect the existence of the destination node within its communication range .Firstly, $\{37,38,39,41\}$ four nodes in order to detect the existence of the destination node within its communication range .If node 39 detects its destination node 53 , and the number of the destination node's children under the limit. Node 39 breaks away from the original parent node 9 and associated with the destination node 53 , and it will receive a new 16-bit short address. If node 38, node 39 and node 41 this three nodes' destination node isn't detected, they keep the existing state of associated. After $\{37,38,39,41\}$ four nodes detection process, $\{9,10\}$ two nodes in order to detect the existence of the destination node within its communication range. After the detection process remaining $\{9,10,37,38,41\}$ five nodes, the paper uses node 9 and node 10 as root to develop subtree, only the subtree root node choose the new parent node to join the non-failing parts of the tree. After joining, all subtree nodes will receive new 16 bit short address. The child node of new recovery node will drill update its own offspring nodes' 16 short address according to the formula (1).

\section{SIMULATION EXPERIMENT}

In order to verify the GHiLow performance, this paper implements simulation experiment on the NS-2.35 simulation platform, and compares with HiLow and E-HiLow in the same simulation environment.NS2 simulation parameters set in Table I.

TABLE I. GHILOW Simulation PARAMETER VALUES

\begin{tabular}{|c|c|}
\hline parameter name & parameter values \\
\hline MAC layer & IEEE802.15.4 \\
number of nodes & 100 \\
size of the simulation environment $/ \mathrm{m}^{*} \mathrm{~m}$ & $100 * 100$ \\
simulation time/s & 600 \\
transmission rate/kpbs & 250 \\
initial energy of nodes $/ \mathrm{J}$ & 20 \\
transmission distance/m & 10 \\
\hline
\end{tabular}

Simulation experiment evaluates the following indicators:

(1) nodes survival ratio: the network node survival rate in the different periods to reflect the survival time of the network.

(2) end-to-end packets delay: according to the improved protocol of average end-to-end packets delay to reflect the performance of the network.

\section{A. Simulation Results and Analysis}

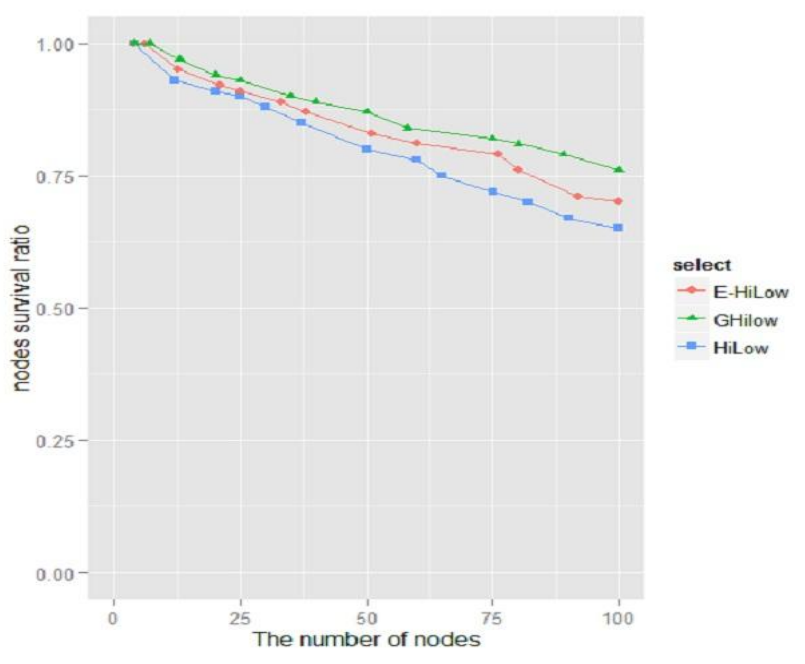

Figure 3. Nodes survival ratio.

It can be seen in figure 3: With the increase of the total number of nodes, the nodes survival rate of E-HiLow, GHiLow and HiLow three protocols all show a down trend. HiLow protocol first generates failed node and the decline range of nodes survival rate curve is the largest. When making the choice of the parent node, compared with HiLow protocol, EHiLow select the node which has the maximum average remainder energy and minimum depth as the parent node and provide the path recovery mechanism, so it later generates failed node and the decline range of nodes survival rate curve is smaller. Of the three protocols GHiLow generates failed node at the latest and the decline range is the smallest. Because in the path recovery of GHiLow protocol, source node may connect directly to destination node so that it effectively reduces the energy consumption of nodes in the communication process. In the parent node selection, GHiLow considers the remainder energy of candidate parent nodes so that the exhausted nodes can appear later; It considers the depth of candidate parent nodes so that the nodes of higher depth can reduce passed nodes in the communication process, reduce the energy consumption effectively and extend the survival time of network. 


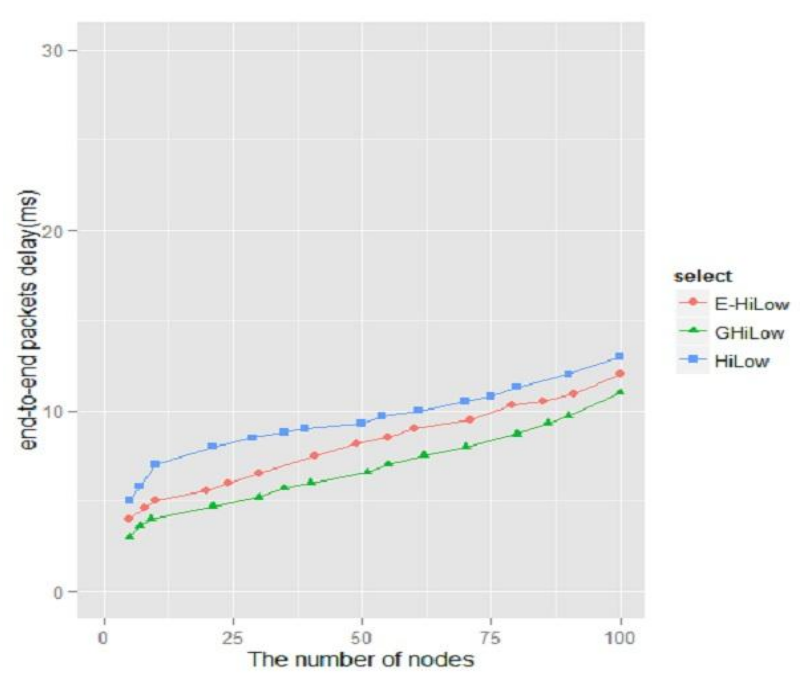

Figure 4. End-to-end packets delay.

It can be seen in figure 4: With the increase of the total number of nodes, the average end-to-end delay of E-HiLow, GHiLow and HiLow three protocols will increase. However, the increased range of HiLow protocol is the largest, because of the load of hierarchical routing tree of HiLow is imbalance. When making the choice of the parent node, E-HiLow select the node which has the maximum average remainder energy and minimum depth as the parent node and provide the path recovery mechanism, its load is more balanced than the load of HiLow, so the increase range is smaller than HiLow. The increase range of GHiLow is the smallest, because in the parent node selection and path recovery, GHiLow considers some factors as node load, remainder energy, node depth, thus the established hierarchical routing tree load is relatively more balanced, and in the path recovery first the descendants of failed nodes find their own destination nodes then doing common path recovery. Compared with other protocols, GHiLow reduce the end-to-end delay effectively and more optimized.

\section{CONCLUSIONS}

6LoWPAN routing protocol is the research hotspot now, HiLow hierarchical routing protocol can meet the 6LoWPAN network low power consumption, low cost characteristics. This paper proposes the GHiLow to improve HiLow hierarchical routing protocol. GHiLow considers more parameters in the parent node selection, and analyzes the candidate parents' different situation. It enhances the performance of the network. What's more, it improves path recovery effectively reducing the energy consumption of the network.

\section{REFERENCES}

[1] Mazzer, Yannis, and Bernard Tourancheau. "Comparisons of 6lowpan implementations on wireless sensor networks." Memory 128.10k (2009): $512 \mathrm{k}$.

[2] Kim, K., et al. "Hierarchical routing over 6LoWPAN (HiLow)." draftdaniel-6Iowpan-hilow-hierarchical-routing-00 (2005).
[3] Kim, Youn-Soo, et al. "Extended tree-based routing algorithm in IPv6enabled wireless sensor networks." Convergence Information Technology, 2007. International Conference on. IEEE, 2007.

[4] Lim, Hun-Jung, and Tai-Myoung Chung. "The bias routing tree avoiding technique for hierarchical routing protocol over 6LoWPAN." INC, IMS and IDC, 2009. NCM'09. Fifth International Joint Conference on. IEEE, 2009.

[5] Chandra, Lingeswari V., et al. "Bias child node association avoidance mechanism for hierarchical routing protocol in 6LoWPAN." Computer Science and Information Technology (ICCSIT), 2010 3rd IEEE International Conference on. Vol. 7, pp. 332-335, IEEE, 2010.

[6] Yue, Li, et al. "E-HiLow: Extended Hierarchical Routing Protocol in 6LoWPAN Wireless Sensor Network." Networking, Architecture and Storage (NAS), 2013 IEEE Eighth International Conference on. IEEE, 2013, pp.8-12.

[7] Nam, Choon-Sung, Hee-Jin Jeong, and Dong-Ryeol Shin. "Extended hierarchical routing over 6LoWPAN." 2008 Fourth International Conference on Networked Computing and Advanced Information Management. Vol. 1, pp. 403-405. 2008.

[8] Ee, Gee Keng, et al. "Path recovery mechanism in 6LoWPAN routing."Computer and Communication Engineering (ICCCE), 2010 International Conference on. IEEE, 2010, pp.1-5. 